\title{
Modeling and Stability Analysis of Linear Actuated Finger
}

\author{
Ellyana Shaira Abd Aziz ${ }^{1 *}$, Muhammad Nizam Kamarudin ${ }^{2}$, Mohd Hafiz Jali ${ }^{1}$ and \\ Rozaimi Ghazali ${ }^{1}$ \\ ${ }^{1}$ Rehabilitation Engineering and Assistive Technology Research Laboratory \\ ${ }^{2}$ Advanced Control Research Laboratory \\ ${ }^{2}$ Robotics and Industrial Automation Research Group (RIA) \\ Centre for Robotics and Industrial Automation (CeRIA) \\ Faculty of Electrical Engineering \\ Universiti Teknikal Malaysia Melaka (UTeM) \\ Malaysia \\ 1*ellyanaaziz@gmail.com; ${ }^{2}$ nizamkamarudin@utem.edu.my, \\ ${ }^{1}$ mohd.hafiz@utem.edu.my; ${ }^{2}$ rozaimi.ghazali@utem.edu.my
}

\begin{abstract}
This paper presents the modeling and validation of a Linear Actuated Finger (LAF) with unknown behavior. In nature, LAF consists of servo, linkage, gear, inertia, stiffness, as well as nonlinearity such as mechanical backlash. The dynamic model is obtained via System Identification (SI) approach. The identification phase exploits the input-output data that represented by the voltage-angle relation of the LAF. By using Jury Stability criterion, the stability of the LAF can be shown without prior knowledge of pole-zero location in the s-plane. Then, simple discrete Proportional-Integral (PI) controller is formulated to validate the controllability as well as to observe the transient and steadystate behavior of the LAF model.
\end{abstract}

Keywords: Linear Actuated Finger (LAF), system identification, stability

\section{Introduction}

Drive systems are fundamental components in industrial process and engineering practice. Among available drive systems, the behavior of Linear Actuated Finger (LAF) system is one of them which widely-used. The behavior of the LAF system under study is unknown. However, prior knowledge indicates that the system consists of servo, linkage, gear, inertia and stiffness, as well as nonlinearities such as mechanical backlash. Unlike wheels or servo-driven arms, linear actuators operate by pushing or pulling along a straight line. There are many potential uses for this, from finely positioning an X-Y table for an automated tool to having the robot servant push a door bell button when human finger gets tired (Shakouri \& Radmanesh, 2009).

Modeling is essential as the first stage of system analysis and control (Ling, Rahmat, Husain, \& Ghazali, 2011). Without prior knowledge of the system dynamics, formulation of control algorithm for such system is impossible, difficult and has become a challenging task. As such, identification of the mathematical model of LAF is the initial problem need to be solved in this research. There are two methods to obtain a mathematical model of a system namely a physical law modeling and system identification. Physical law modeling is demanding expert knowledge about the system to be modeled. However, such knowledge about the system is hard to obtain. On the other hand, system identification has many advantages that overcome the shortcoming of physical law modeling approach. System identification requires no prior knowledge about the system behavior (Khamis, Kamarudin, Lada, Saifuzam, Hanafi, \& Nazmi). By obtaining the input-output relation of 
the system, the mathematical model can be formulated. As such, system identification method is always known as "black box" identification as shown in Figure 1.

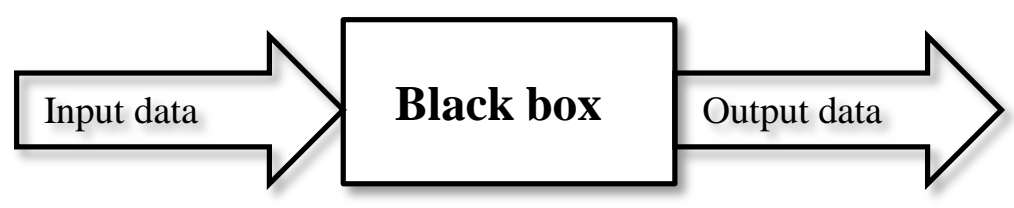

Figure 1. Black Box Modeling

The system identification (Li Y. J., 2003) is to determine a model that is equivalent to the identified system from a group of given models based on the input and output data. The purpose of system identification is to establish a mathematical model that can resolve practical problems. System identification uses simple data has been a major studies in various fields. Despite being relatively mature and well-developed, conventional system identification still has many limitations (Li \& Han, 2004). Although such shortcomings of conventional system identification methods may be solved to a certain extent by some new methods for system identification, such as the system identifications based on neural network and based on genetic algorithm, these methods typically fail to properly handle system identification when then structure and parameters are completely unknown. With regard to this problem, authors in (Liu \& Tang, 2007) offer a new method or identifying system model based on typical models and sample data, thus the problem of system identification is converted into a problem of combinatorial optimization.

System identification includes linear system identification and nonlinear system identification (Li. F., 2013). Although linear models are simple and able to be implemented in real-time, there are minimal studies on linear models as most of the previous studies used neural networks (Santina, Allen, \& G, 1996).

Such that, the model of LAF is depicted in discrete time system for its advantages over continuous time representation, such as;

1. Low cost, low weight, and low power consumption.

2.Zero drift of system parameters despite wide variations in temperature, humidity, and component aging.

3. High accuracy

4. High reliability and ease of making software and design changes.

Discrete-time signals are defined only for discrete instants of time, usually at evenly spaced time steps. A discrete system is said to be linear if it satisfies the superposition principle. Any linear combination of inputs produces the same linear combination of corresponding output components. A discrete-time system is step invariant if its properties do not change with time step. Any time shift of the inputs produces an equal time shift of every corresponding output signal (Santina, Allen, \& G, 1996).

Generally speaking, the basic requirement for automatic control systems is that the system must be stable. Next, the transient performance of the system should meet the requirement of the production process of the transient performance. The third, the stability error of the system should meet the production process requirement. The stability of the system is not only important for the performance of control system, but it is mandatory for the system to work properly. The control system is more or less influenced by external and internal factors of disturbance in actual operation. For instance, load and energy fluctuations, changes in system parameters, environmental conditions and environmental changes. If the system is unstable, perturbation may diverge that makes the perturbed states away from the origin. A well designed controller may then force the perturbed states to the origin. Thus, how to analyze the stability of the system and to ensure system stability is the basic task of the automatic control theory (Z, J, \& X, 2012). 
This paper is structured as follows: a system modeling experimental setup and simulation, system identification, and transfer function modeling are discussed in Section II. Section III discusses the result and analysis of the system based on stability analysis and step responses. Finally, Section IV draws the conclusions.

\section{System Modeling}

\subsection{Experimental Setup}

The experimental setup of the LAF system contains a few main parts; a laptop, motor driver, NI MyRio and the LAF plant as shown in Figure 1. Whereas Figure 2 shows the configuration set up that facilitate identification process. An input signal is generated by using LabVIEW ${ }^{\circledR}$ software platform and being injected to the motor driver through NI MyRio Module. The motor driver is a 4 channels DC motor driver that specially designed to drive four DC brush motors. With minimum interfacing apparatus, the board is ready to drive the motor with forward-reverse direction, start-stop operation and speed control feature. The position of the LAF angle is measured by using the conventional protector. Experiment is commenced by stimulating the LAF with $5.2 \mathrm{~V}$ for $46^{\circ}$ angle of position. The procedure is depicted in the flow chart in Figure 3.

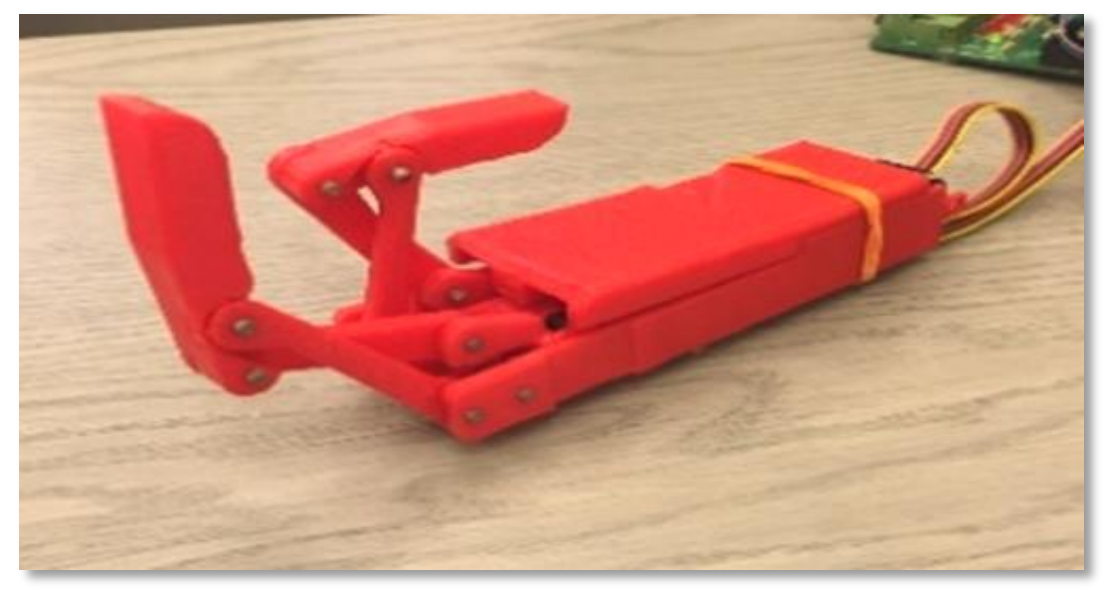

Figure 1. Linear Actuated Finger (LAF)

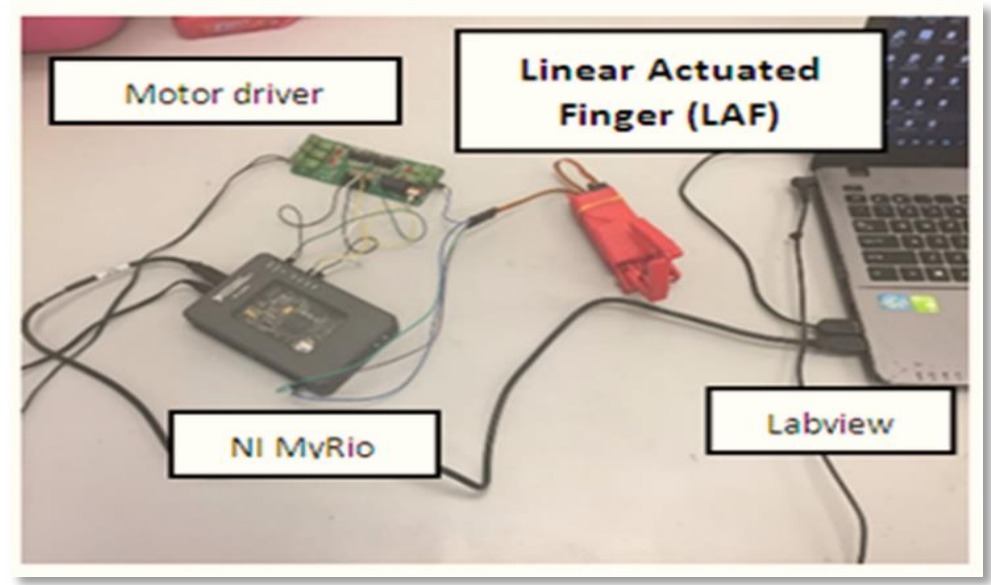

Figure 2. Experiment Setup and Configuration 


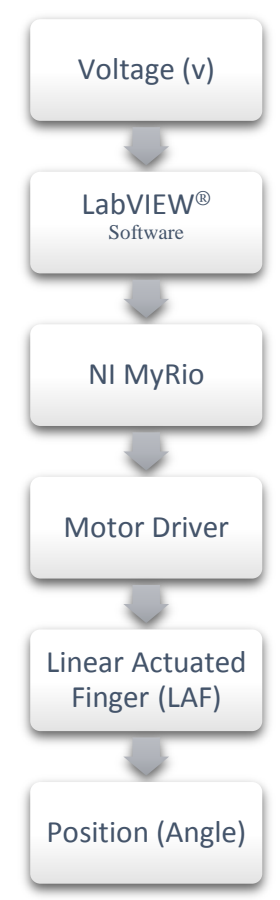

Figure 3. Data Collection Flowchart

\subsection{Simulation}

Before system identification process takes place, the model of the LAF is developed and an open loop system is applied. The converter is used to convert a voltage to position based on the movement of the linear motor. Based on Equation (1), the voltage is represented as motor input. Motor input parameter is set on analogue parameter.

From the input-output data acquisition model in Figure 4, the parameter for the controller can be set. The set point is fixed at $5 \mathrm{~V}$. The speed of the LAF is varied by voltage changes.

$$
y=m x+c
$$

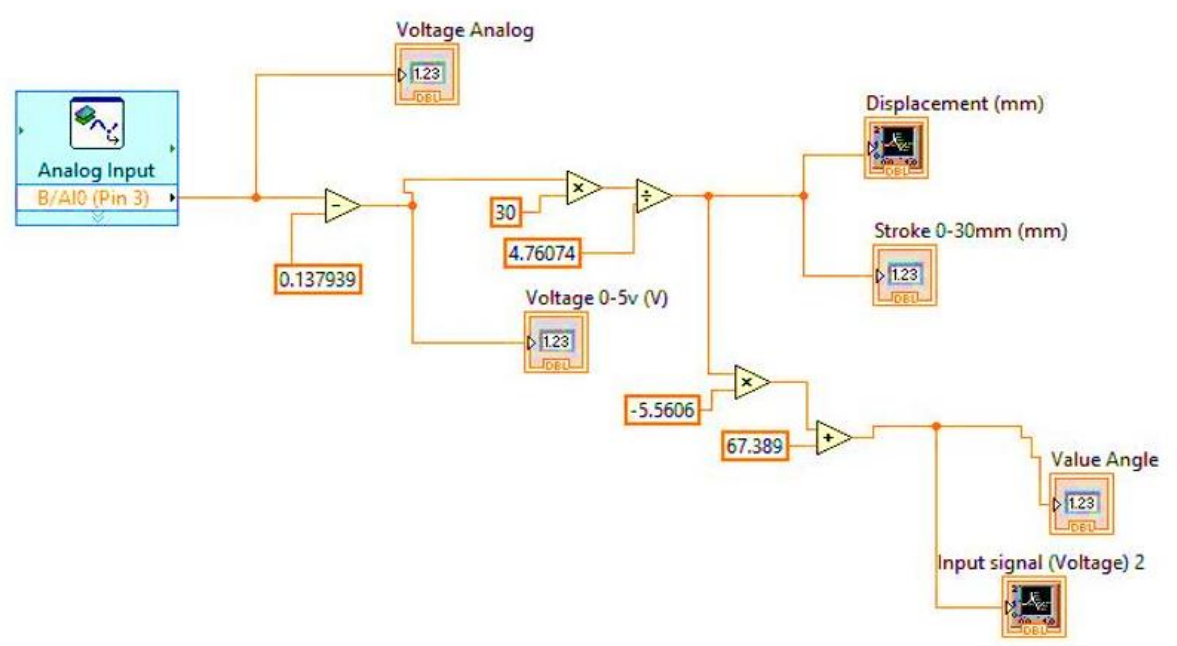

Figure 4. LAF Input-Output Data Acquisition Model 
As such, linear relationship between displacement $(d)$ due to input voltage and finger angle $(\theta)$ is depicted as shown in Equation (2).

$$
\theta=-5.5606 d+67.389
$$

The mapping of the finger's angle can be taken on the reference point on the left side middle phalanx when the voltage feedback controls the displacement stroke motor. Table 1 shows the mapping positioning angle of finger when the motor is operated. The relationship between the displacement and positioning parameter generate the mathematical gradient equation in order to convert the displacement into the angle of finger.

Based on Figure 5, the angle of position finger where the left middle phalanx as reference point shows that the maximum angle is $67.389^{\circ}$ with gradient -5.5606 . When the finger is totally $90^{\circ}$ degree angle, the angle of left middle phalanx reached to the maximum point of angle.

\section{Table 1. The Relationship between Displacement and Positioning Angle}

\begin{tabular}{|c|c|}
\hline Displacement $(\mathbf{m m})$ & Positioning $(\boldsymbol{\theta})$ \\
\hline 11.6308 & $0^{\circ}$ \\
\hline 9.20001 & $15^{\circ}$ \\
\hline 7.53077 & $30^{\circ}$ \\
\hline 5.24616 & $40^{\circ}$ \\
\hline 3.11539 & $50^{\circ}$ \\
\hline 0.0230797 & $60^{\circ}$ \\
\hline
\end{tabular}

Angle

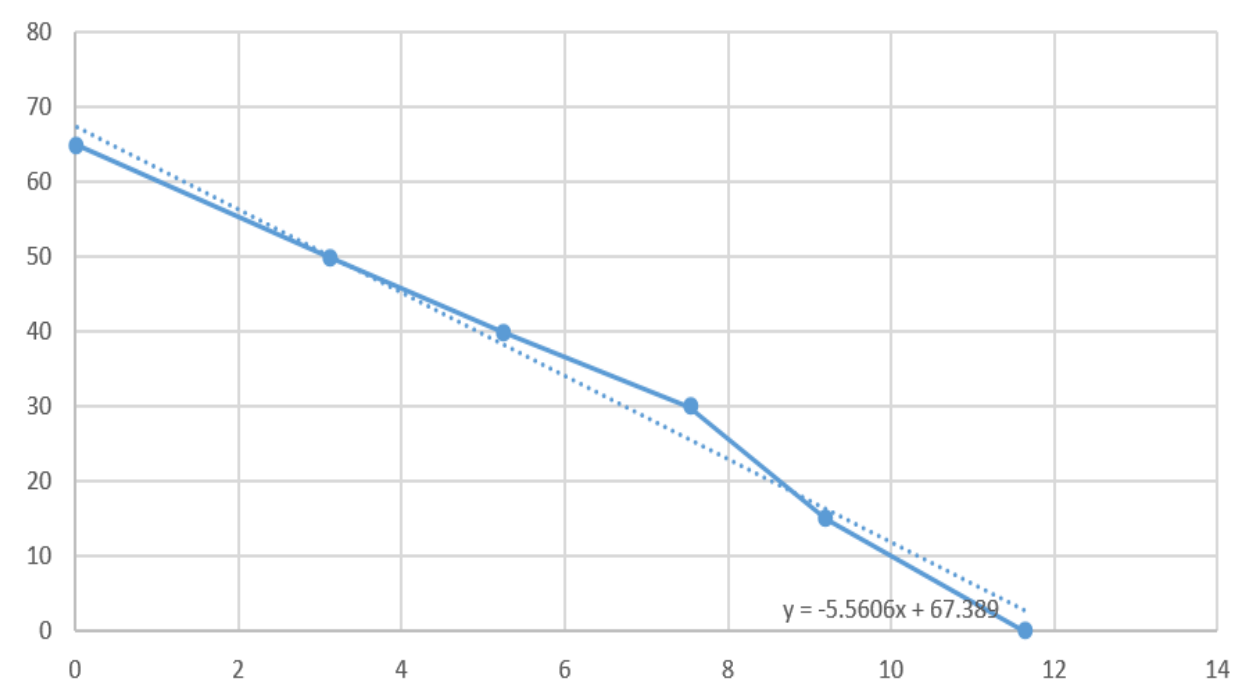

Figure 5. The Relationship between the Angle and Displacement

\subsection{System Identification}

System identification process for LAF is depicted in Figure 6. The basic of the systems consists of four main components. These components are the plant to be controlled, followed by reference model that is needed to generate desired closed loop for output 
response. The controller is time varying and whose coefficients are adjusted by adaptive mechanism. Lastly, an adaptive mechanism that uses 'error' (the difference between the plant and desired model output) in order to produce controller coefficient.

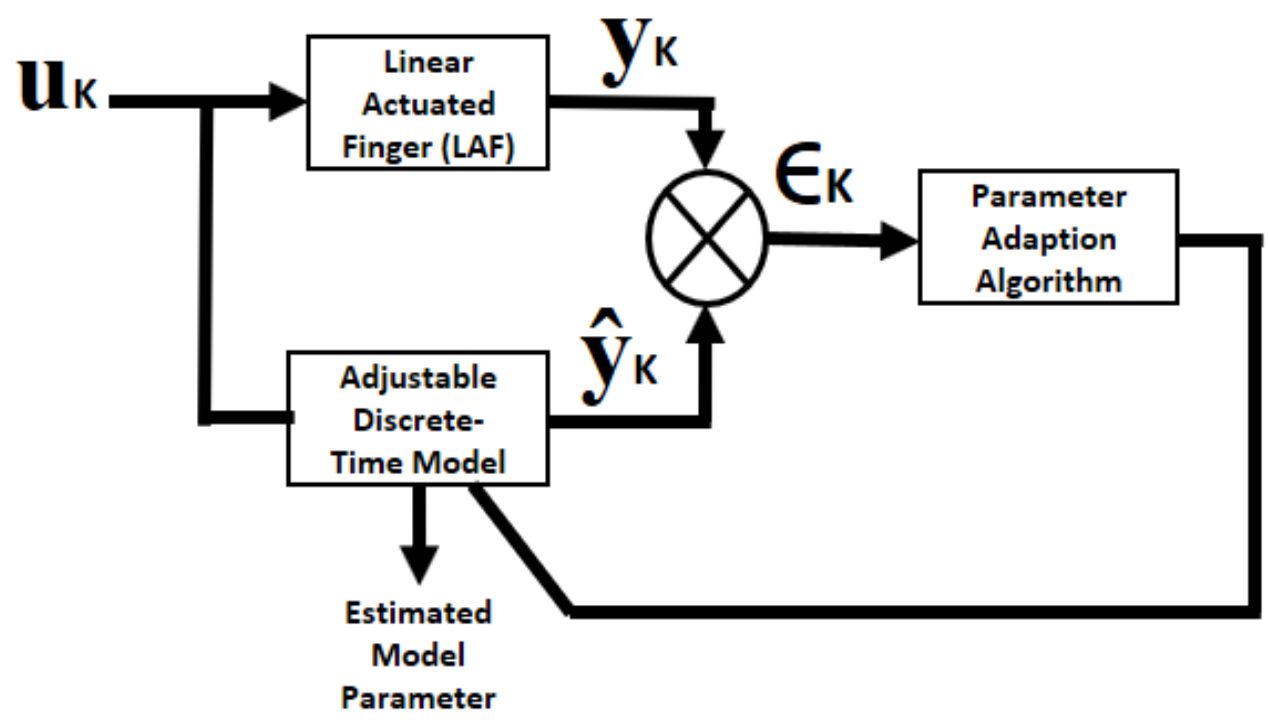

Figure 6. Linear Actuated Finger (LAF) System Identification Process

From Figure 6, the terms $u(k)$ and $y(k)$ are the input and output signals respectively. From the system identification process, important criteria such as best fits, residual analysis for model correlation (Figure 8), pole-zero location (Figure 9), and frequency response (Figure 10) is recorded in order to estimate the LAF model. Figure 7 shows the model output yields 84.47 best fits.

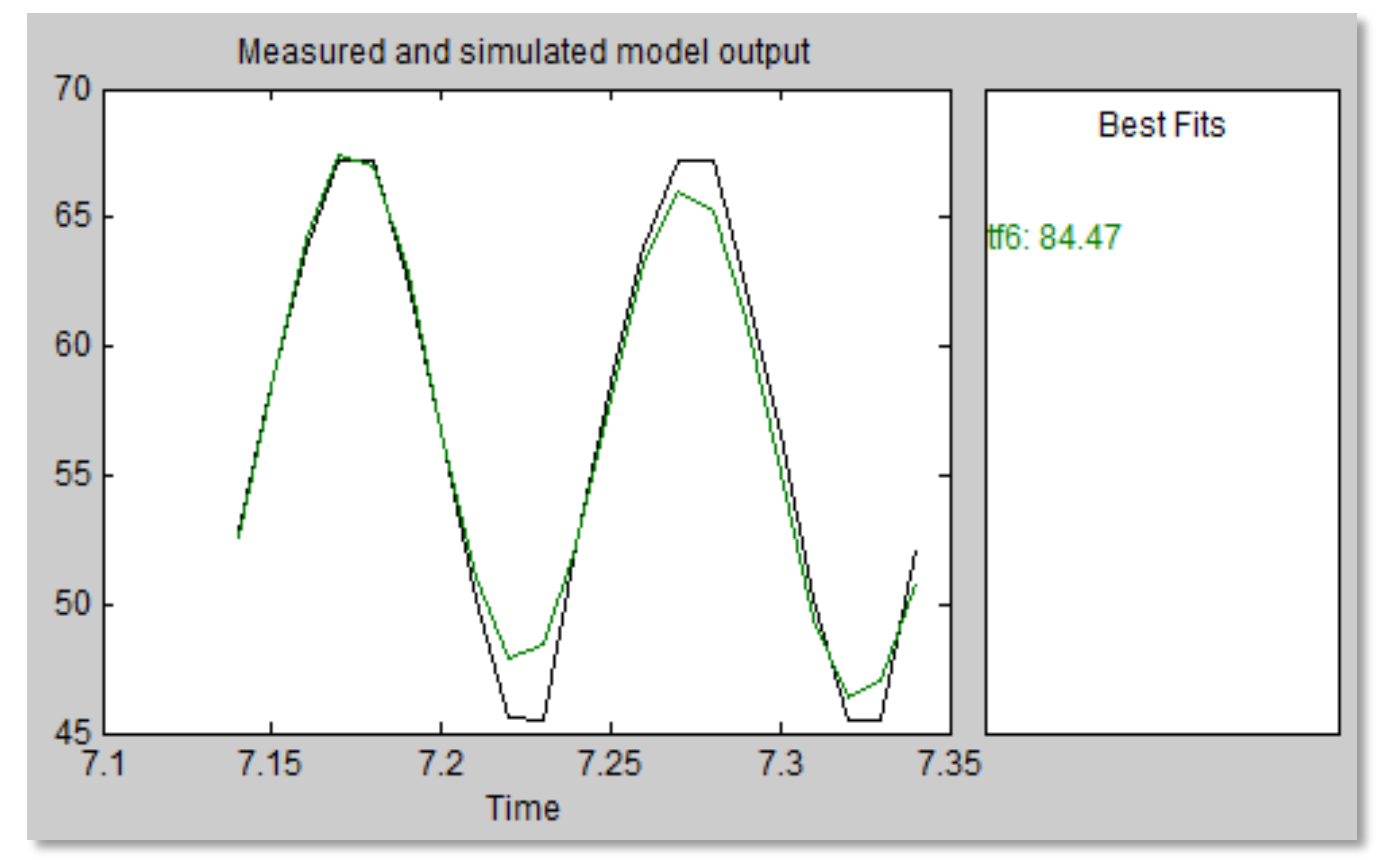

Figure 7. Measured and Simulated Model Output Yields Best Fits 84.47 

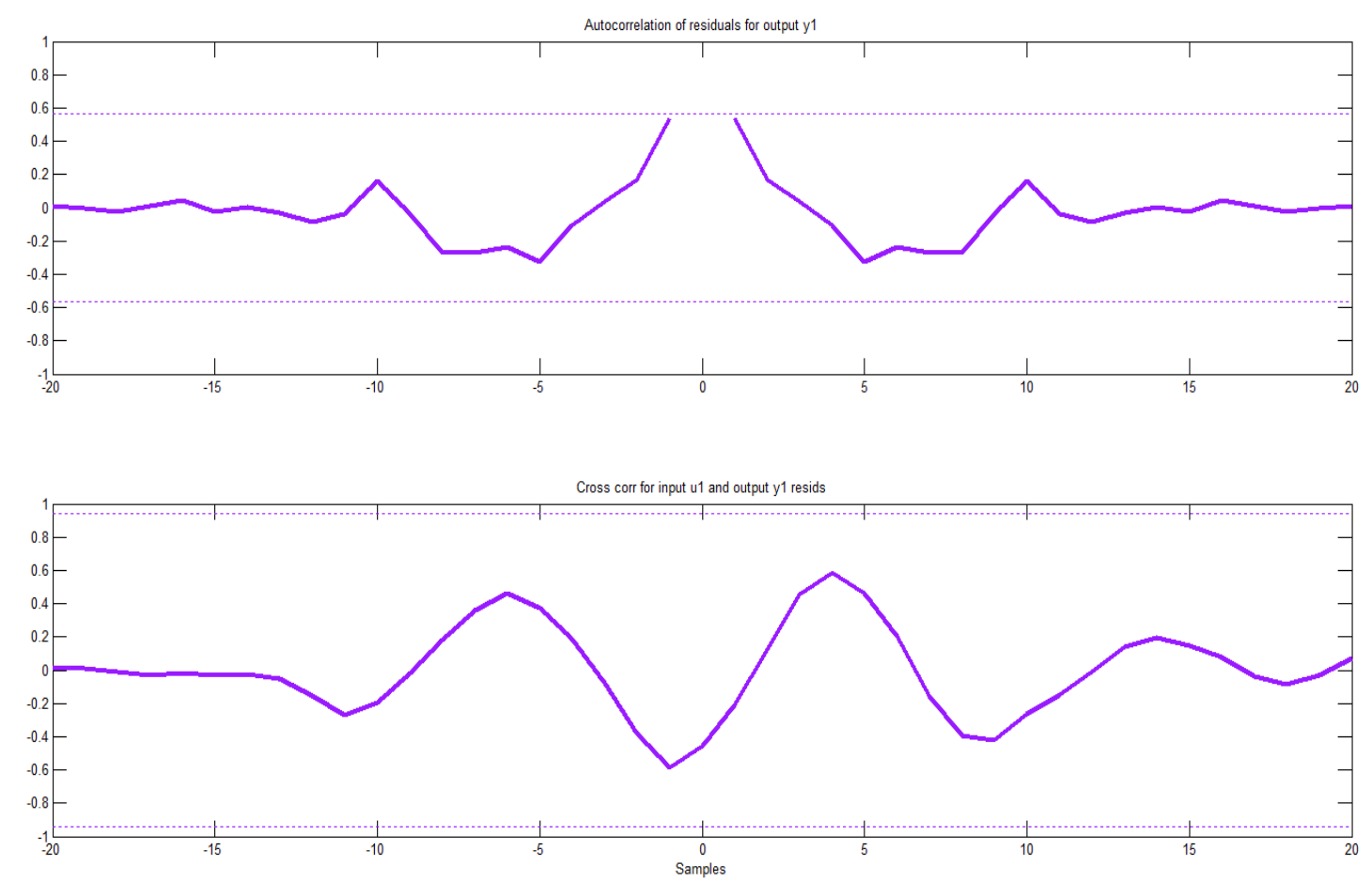

Figure 8. Model Correlation

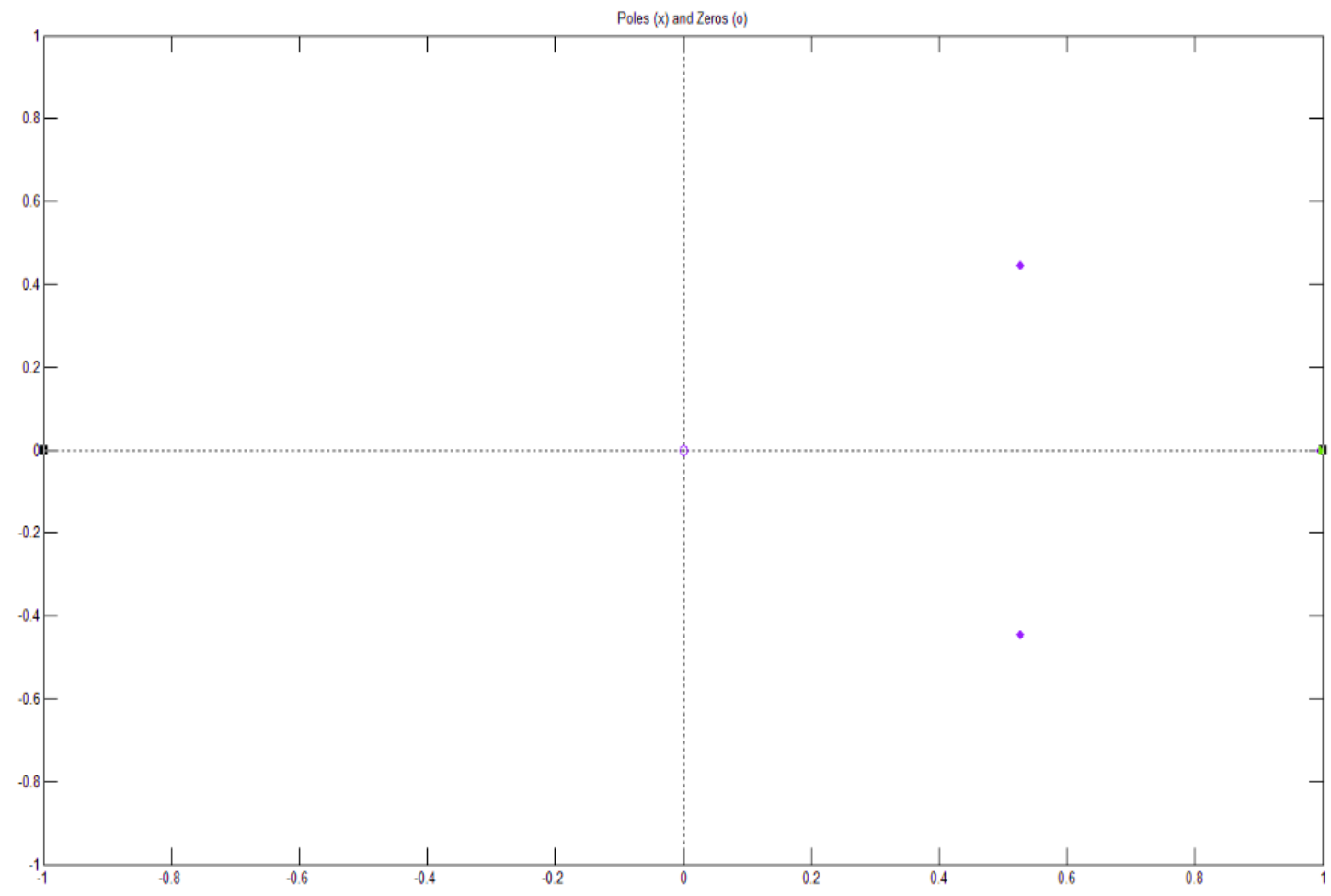

Figure 9. Pole-zero 

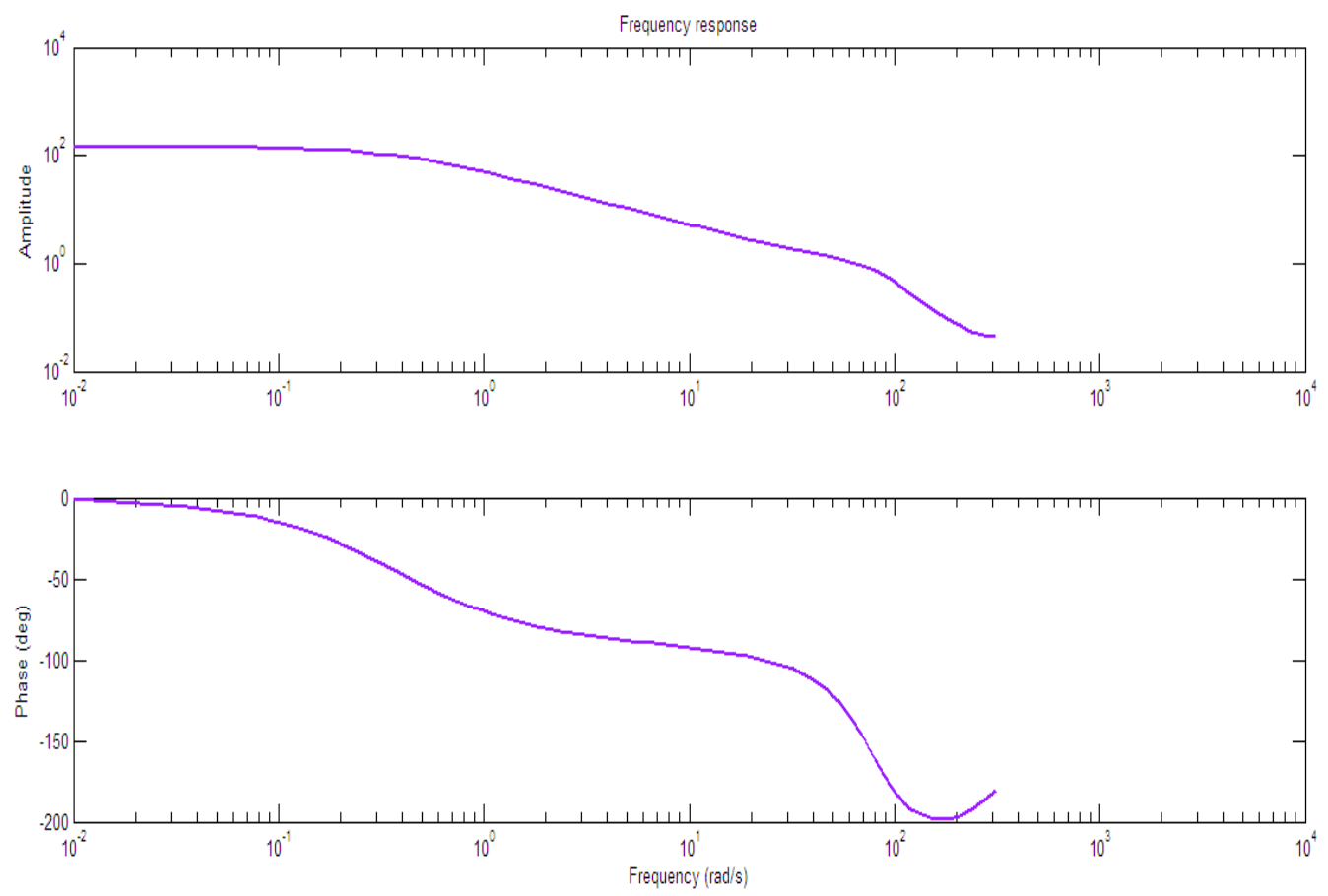

Figure 10. Frequency Response

\subsection{The LAF Model and Dynamic}

The LAF model and dynamic is represented in discrete-time in the form of block diagram of Figure 11.

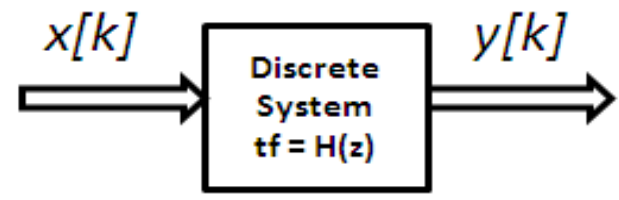

Figure 11. Discrete Time System, With Transfer Function H(z)

Signal $x(k)$ and $y(k)$ represent the input and output data respectively. $H(z)$ represents the Laplace transform of the system in discrete time.

The input-output data collected from the plant is the voltage and position, as shown in Figure 12 and Figure 13 respectively. From the frequencies given to the input signal, the models that can be obtained are limited to third order system only. Higher order may produces unstable output. In this case, the third order model presents the nearest model of actual plant. The output signal of the LAF is the position of the LAF as shown in Figure 13.

The input and output signals are divide into two groups, 295 to 322 samples and 615 to 635 samples. The first group of the input-output signals is used to obtain the plant model and the second group is used for validation. 


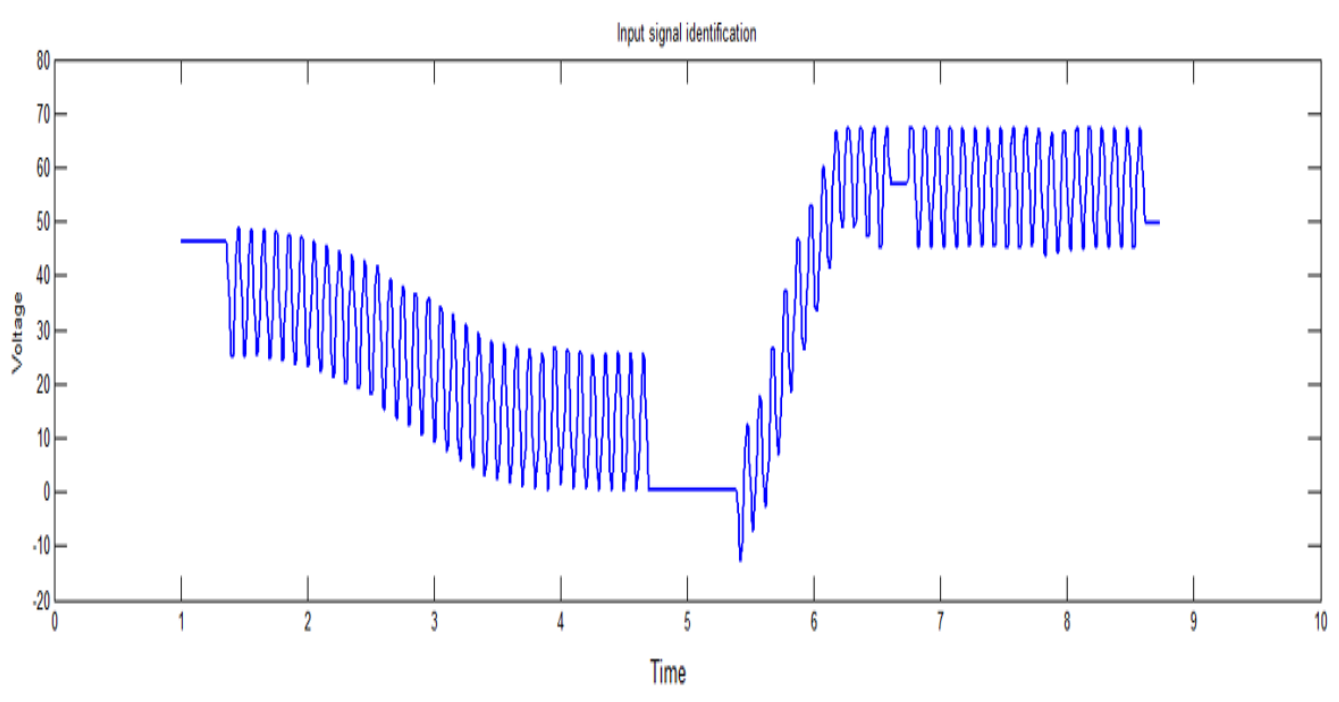

Figure 12. Input Signal

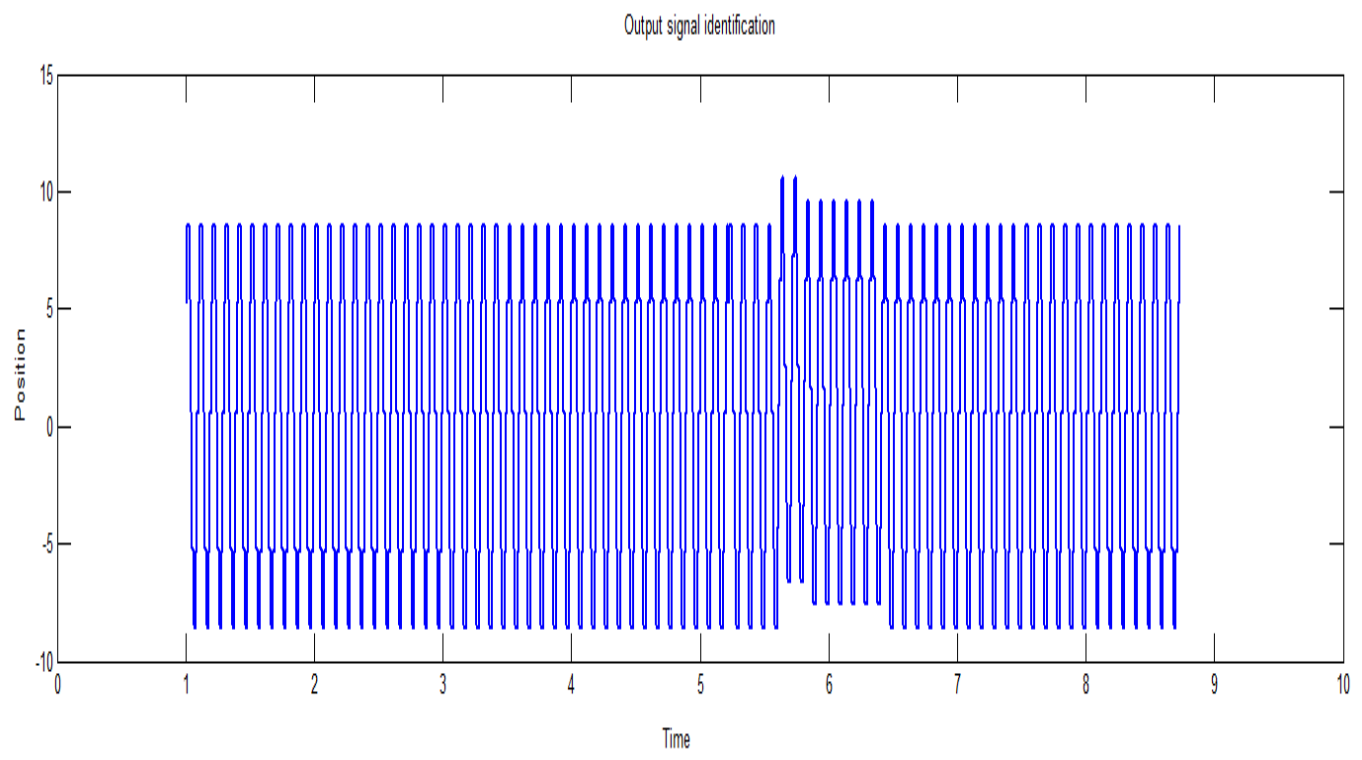

Figure 13. Output Signal

\section{Result and Analysis}

The identification tool in MATLAB ${ }^{\circledR}$ software generates discrete time polynomial shown in Equation (3). Equation (4) provides information on the mathematical model of the LAF system in discrete time domain whereas; Equation (5) shows the transfer function of the system in frequency domain.

$$
\begin{gathered}
G(z)=\frac{0.2223 z^{-1}}{1-1.831 z^{-1}+1.53 z^{-2}-0.4754 z^{-3}} \\
G(z)=\frac{0.2223 z^{2}}{z^{3}-1.831 z^{2}+1.53 z-0.4754}
\end{gathered}
$$




$$
G(s)=\frac{0.9471 s^{2}+31.1 s+332}{s^{3}+7.436 s^{2}+62.95 s+2.39}
$$

\subsection{Stability Analysis}

Jury stability criterion is performed to observe the stability of the system. By using Jury stability criterion, the information about unstable or stable roots is obtained. To perform Jury stability criterion, the polynomial denominator of the system dynamics is considered, as in Theorem 1. Corollary to Theorem 1, the stability of the LAF is concluded in Proposition 1.

Theorem 1 (Keel \& Bhattacharayya, 1999)

Let the polynomial of a discrete linear system be

$$
A(z)=a_{0} z^{n}+a_{1} z^{n-1 n}+\ldots+a_{n-1} z^{n}+a_{0} z^{n}+a_{n}
$$

Table 2. Jury Stability Criterion Table

\begin{tabular}{|c|c|c|c|c|c|c|c|}
\hline $\boldsymbol{R o w}$ & $\boldsymbol{z}_{\mathbf{0}}$ & $\boldsymbol{z}_{\mathbf{1}}$ & $\boldsymbol{z}_{\mathbf{2}}$ & $\cdots$ & $\boldsymbol{z}_{\boldsymbol{n}-\mathbf{2}}$ & $\boldsymbol{z}_{\boldsymbol{n}-\mathbf{1}}$ & $\boldsymbol{z}_{\boldsymbol{n}}$ \\
\hline $\boldsymbol{1}$ & $a_{n}$ & $a_{n-1}$ & $a_{n-2}$ & $\cdots$ & $A_{1}$ & $a_{1}$ & $a_{0}$ \\
\hline $\mathbf{2}$ & $a_{0}$ & $a_{1}$ & $a_{2}$ & $\ldots$ & $a_{n-2}$ & $a_{n-1}$ & $a_{n}$ \\
\hline $\mathbf{3}$ & $b_{n-1}$ & $b_{n-2}$ & $b_{n-3}$ & $\cdots$ & $B_{1}$ & $b_{0}$ & \\
\hline $\mathbf{2}_{\boldsymbol{n}-\mathbf{5}}$ & $p_{3}$ & $p_{2}$ & $p_{1}$ & & & & \\
\hline $\mathbf{2}_{\boldsymbol{n}-\mathbf{4}}$ & $p_{0}$ & $p_{1}$ & $p_{2}$ & & & & \\
\hline $\mathbf{2}_{\boldsymbol{n}-\mathbf{3}}$ & $q_{2}$ & $q_{1}$ & $q_{0}$ & & & & \\
\hline
\end{tabular}

Then, the tabulation of coefficient in Table 2 is observed such that;

$$
\left|a_{n}\right|<\left|a_{0}\right|
$$

And

$$
A(1)>0
$$

and

$$
A(-1)\left\{\begin{array}{l}
>0 \text { for } n \text { even } \\
<0 \text { for } n \text { odd }
\end{array}\right.
$$

and 


$$
\begin{gathered}
\left|b_{n-1}\right|>\left|b_{0}\right| \\
\left|c_{n-1}\right|>\left|c_{0}\right| \\
\vdots \\
\left|a_{n-1}\right|>\left|a_{0}\right|
\end{gathered} \mid A(Z) \text { order }>2
$$

If and only if, all requirement in (7), (8), (9) and (10) are fulfilled, then the system with polynomial $\mathrm{A}(\mathrm{z})$ in $(6)$ is stable.

\section{Proposition 1}

A discrete Linear actuated finger model in equation (4) is stable in a sense of Jury stability criterion.

Proof

Consider the polynomial of the system as

$$
z^{3}-1.8321 z^{2}+1.53 z-0.4754
$$

The polynomial in equation (11) shows that the system is $3^{\text {rd }}$-order. As such, a table of $2 \mathrm{n}-3$ for $\mathrm{n}=3$ is constructed as presented in Table 3 .

Table 3. Proposition Jury Stability Criterion Table

\begin{tabular}{|c|c|c|c|c|}
\hline $\boldsymbol{R o w}$ & $\mathbf{z}_{\mathbf{0}}$ & $\mathbf{z}_{\mathbf{1}}$ & $\mathbf{z}_{\mathbf{2}}$ & $\mathbf{z}_{\mathbf{3}}$ \\
\hline $\mathbf{1}$ & -0.4754 & 1.53 & -1.831 & 1 \\
\hline $\mathbf{2}$ & 1 & -1.831 & 1.53 & -0.4754 \\
\hline $\mathbf{3}$ & -0.7740 & 1.1036 & -0.60 & \\
\hline
\end{tabular}

From Table 3, it is shown that all requirements in Equation (7), Equation (8), Equation (9) and Equation (10) are fulfilled. Therefore, the LAF system in Equation (4) is stable.

\subsection{Step Response}

Preliminarily, Proportional-Integral (PI) controller is formulated in order to obtain the tracking control of the position angel $\theta$. The PI parameter is tabulated in Table 4 .

Table 4. PI Parameter

\begin{tabular}{|c|c|c|}
\hline Parameters & P & I \\
\hline Optimized value & 1 & 0.01 \\
\hline
\end{tabular}

The relationship between the measured and stimulated input-output data are plotted as frequency response to identified the angle position (angle) of the system.

Figure 14 shows the system response to a step input. The system rises at 0.221 . At 0.4 seconds, the response reaches peak amplitude at 1.26 with $26.9 \%$ overshoot. The oscillation settles at 2.81 seconds. The steady-state error is recorded at 0.993 which is $0.7 \%$ deviation from the demanded step input. 


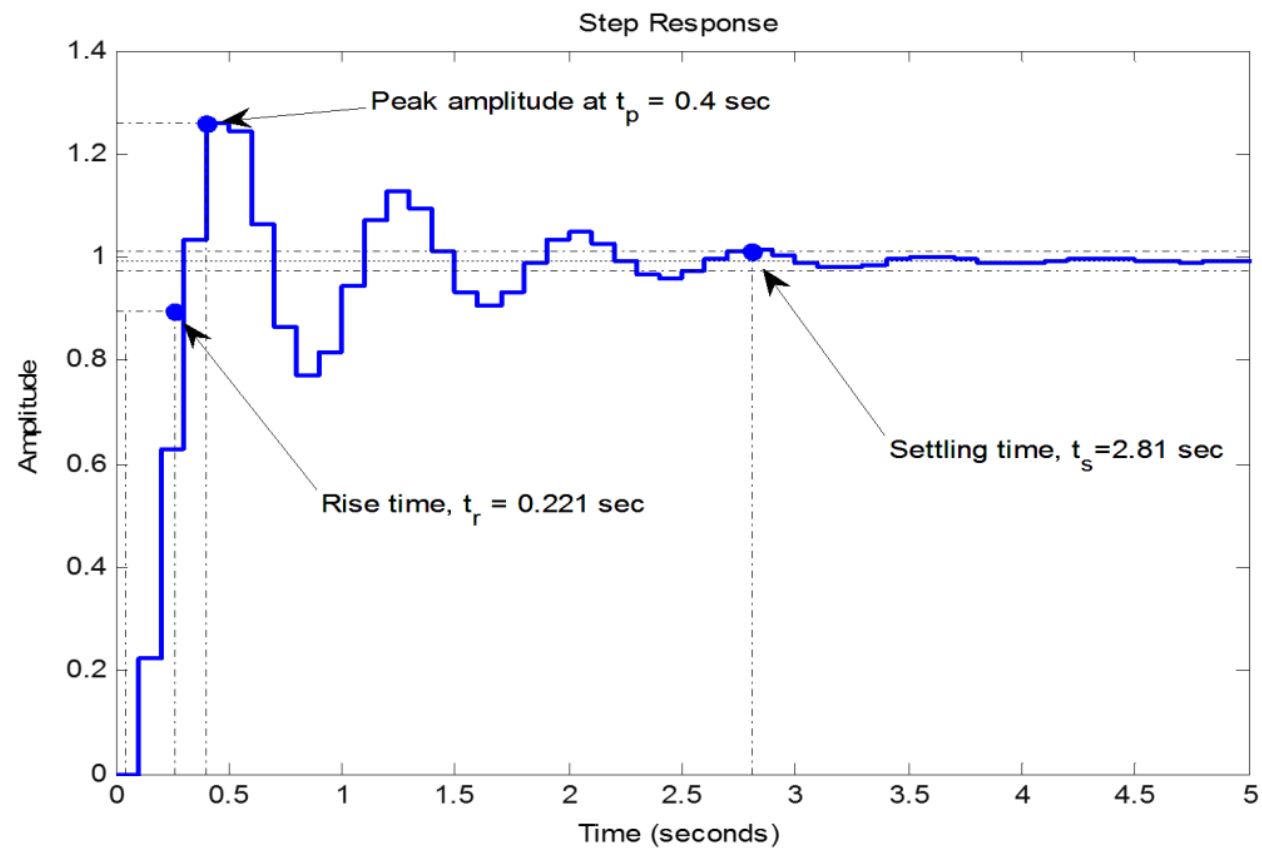

Figure 14. System Response to a Step Input with PI Controller

\section{Conclusion}

In this experiment, the mathematical model and dynamics of the LAF is successfully obtained by using system identification approach. The use of system identification relaxes the necessary of using complex frequency domain and time domain modelling that normally require computational burden. With $84.47 \%$ best-fit, the system appeared as $3^{r d}$-order dynamics with all poles are located inside the unit circle of the $z$-plane. The stability of the system is then guaranteed by Jury stability criterion. To validate the controllability of the LAF system, simple two-term PI controller is designed in order to achieve the position tracking. The proposed closed-loop control method has been implemented in MATLAB $^{\circledR}$ with SIMULINK ${ }^{\circledR}$ toolbox that facilitates the auto-tuning process where the optimum parameters are chosen based on the system speed, transient and steady state response.

\section{Acknowledgement}

The authors would like to thank to MyBrain UTeM scholarship provided by Universiti Teknikal Malaysia Melaka. This research experimental set-up is funded by research grant no. RAGS/1/2015/TK0/FKE/03/B00097.

\section{References}

[1] L. H.Keel and S. P. Bhattacharayya, "A New Proof of the Jury Test”, Automatica, vol. 35, (1999), pp. 252-258.

[2] A.Khamis, M. N.Kamarudin, M. Y.Lada, M. Saifuzam, F. Hanafi and A.Nazmi, (n.d.), "Identification and Simulation of DC-DC Boost Converter for Charging Up PV Volatge for 24-volts Battery", The Asis-Pasific Symposium and Applied Electromagnetics.

[3] Y. J. Li, "Theory and Application of System Identification", Beijing: National Defence Industry Press, (2003)..

[4] Y. X. Li and Z. G. Han, "Advances in nonlinear system identification”, Techniques of Automation \& Application, vol. 23, no. 10, (2004), pp. 5-7.

[5] F.Li and L. P., "The Research Survey of System Identification Method", Fifth International Conference on Human Intelligent Human-Machine Systems and Cybernatics, (2013), pp. 397-401. 
[6] T. G. Ling, M. F. Rahmat, A. R. Husain and R. Ghazali, "System Identification of Electro-Hydraulic Actuator Servo System", 4th International Conference on Mechatronics (ICOM). Kuala Lumpur Malaysia, (2011).

[7] S. A. Liu and F. Tang, "Study on the system identification method based on genetic algorithm", System Engineering Theory and Practice, vol. 16, no. 3, (2007), pp. 134-139.

[8] M. S. Santina, S. R.Allen and G, H. H., "Discrete-Time Systems", The Control Handbook, (1996), pp. 239-251.

[9] G. H. Shakouri and H. R. Radmanesh, "Identification of a continuous time nonlinear state space model for the external power system equivalent by neural network", Electrical Power and Energy Systems, vol. 31, (2009), pp. 334-344.

[10] Z. Y., J. L. and X. W., "The Stability Analysis and Simulation of Linear Systems via LMI", IEEE Symposium on Electrical \& Electronics Engineering (EEESYM), (2012), pp. 532-535.

\section{Authors}

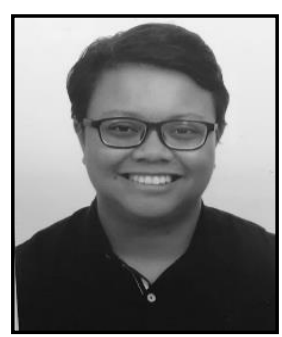

Ellyana Shaira Binti Abd Aziz, he received his B. Eng. Tech. degree in Electronic Engineering (Industrial Electronic) from Universiti Teknikal Malaysia Melaka (UTeM) in 2015. Currently, she pursues her study in Master of Science on Electrical Engineering, UTeM.

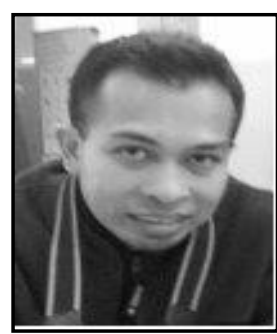

Muhammad Nizam Kamarudin, he received the B. Eng (Hons.) Electrical from the Universiti Teknologi MARA, Malaysia (UiTM) in 2002, M.Sc. in Automation and Control from the University of Newcastle Upon Tyne, United Kingdom in 2007 and Ph. D in Electrical Engineering from Universiti Teknologi Malaysia (UTM) in 2015. He is currently with the Universiti Teknikal Malaysia Melaka (UTeM). He is the member of the Board of Engineers, Malaysia and Institute of Engineers, Malaysia. His research interests include nonlinear controls and robust control systems. Before joining UTeM, he worked as a technical engineer at the magnetron department of Samsung Electronics Malaysia.

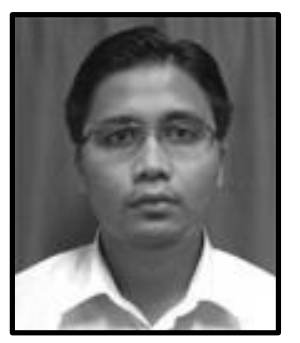

Mohd Hafiz bin Jali. he received the B.Sc. degree in electrical and electronic engineering with first class honors from MARA University of Technology, Malaysia in 2007 and completed his Master engineering studies with distinction in Industrial Electronics and Control at University of Malaya, Malaysia. He was with automotive company, Proton Sdn. Bhd from 2007 to 2010 as a Test Engineer under the Complete Vehicle Testing (CVT) department. In 2012, he was appointed as a Lecturer at Universiti Teknikal Malaysia Melaka, Malaysia. His research interests are in the general area of control, instrumentation and automation.

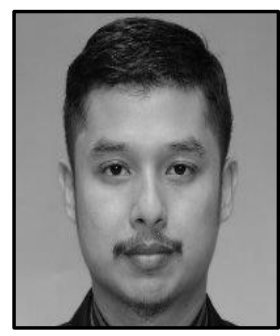

Rozaimi Ghazali, he received his B.Eng degree in Electrical Engineering (instrumentation and Control) from Universiti Teknologi Malaysia (UTM), in 2008. He received the Ph. D in Electrical Engineering also from UTM, in 2013. Currently, he is a senior lecturer at Universiti Teknikal Malaysia Melaka (UTeM) and his interests are in system identification and robust controller design. 
International Journal of Control and Automation

Vol. 10, No. 1 (2017) 